\title{
Mechanical Properties of Silica Glass Predicted by a Pair-wise Potential in Molecular Dynamics Simulations
}

\author{
Siddharth Sundararaman ${ }^{\mathrm{a}}$, Wai-Yim Ching ${ }^{\mathrm{b}}$, Liping Huang ${ }^{\mathrm{a},+}$ \\ ${ }^{a}$ Department of Materials Science and Engineering, Rensselaer Polytechnic Institute, Troy, NY \\ 12180 \\ ${ }^{\mathrm{b}}$ Department of Physics, University of Missouri-Kansas City, Kansas City, MO 64110
}

\begin{abstract}
Pair-wise BKS potential for silica glass was modified by changing the short-range cutoff to improve the prediction of mechanical properties. Classical molecular dynamic (MD) simulations were carried out by using this modified potential and better prediction of mechanical properties at ambient conditions and their changes with pressure at room temperature were shown. It was further observed that there was no major change in structural properties when different shortrange cutoffs were used to relax the glass. To better understand the contribution of structure to the mechanical properties, samples quenched under various cooling rates and one structure optimized in first principles calculations were used. It was seen that the improvement of structure has a less effect than improving the interaction potential in determining the mechanical properties of BKS silica glass. The strength and weakness of the BKS potential for silica glass was discussed.
\end{abstract}

${ }^{\dagger}$ Corresponding author. E-mail address: huangL5@ rpi.edu (L. Huang). 


\section{Introduction}

One of the most commonly used potentials for silica glass is the pair-wise BKS potential [1]. This potential was parameterized to fit ab-intio data of an $\mathrm{H}_{2} \mathrm{SiO}_{4}$ cluster with added constraints to predict the structural parameters and elastic constants of $\alpha$-quartz correctly. Its popularity is mainly due to its ability to predict a wide range of properties for silica glass and its crystalline forms, with reasonable accuracy for such a simple potential form [2-10]. However, this potential form has a few deficiencies, for example, its inability to reproduce the equation of state of liquid silica [11], high concentration of surface defects generated by this potential as compared to CarParinello molecular dynamics data [12], and thermodynamic instability of BKS cristobalite and tridymite silica [2]. The compressibility anomaly under pressure for silica glass where it initially becomes softer upon hydrostatic compression with a minimum in bulk modulus at about $\sim 2-3$ $\mathrm{GPa}$ is a well observed phenomenon in experiments [13-16]. Although a Wolf truncated version of the BKS potential can qualitatively describe the initial softening of silica glass under pressure, it tends to overestimate the elastic moduli such as bulk modulus at ambient conditions, and also predict the bulk modulus minimum at much higher pressures (8-9 GPa) [17].

The main objective of this work is to improve the prediction of mechanical properties and their changes with pressure by varying the short-range cutoff of the BKS potential. The effect of structure on the prediction of mechanical properties was also studied by quenching glass under different cooling rates and by using an ab-initio structure [18-21]. Our study illustrated some of the factors and their relative importance in the correct prediction of mechanical properties of silica glass, and pointed out the strength and weakness of the BKS potential.

\section{Computational Detail}

\subsection{Simulation method}

Molecular dynamic (MD) simulations were performed in the large-scale atomic/molecular massively parallel simulator (LAMMPS [22] package using a modified BKS potential [1] as proposed by Vollmayr et al. [6]. The pairwise interatomic potential is of the form as shown in Eq. (1).

$$
\phi_{i}=\frac{q_{i} q_{j} e^{2}}{r_{i j}}+A_{i j} e^{-B_{i j} r_{i j}}-\frac{C_{i j}}{r_{i j}^{6}}
$$

where $q_{i}$ is the partial charge on atom $i$, and $A, B$ and $C$ are parameters whose values are given in Table. 1. 
Table 1 Parameters of the BKS potential [1].

\begin{tabular}{|l|l|l|l|}
\hline $\mathrm{i}-\mathrm{j}$ & $\mathrm{A}_{\mathrm{ij}}(\mathrm{eV})$ & $\mathrm{B}_{\mathrm{ij}}\left(\AA^{-1}\right)$ & $\mathrm{C}_{\mathrm{ij}}\left(\mathrm{eV} \cdot \AA^{6}\right)$ \\
\hline $\mathrm{O}-\mathrm{O}$ & 1388.773 & 2.76 & 175.0 \\
\hline $\mathrm{O}-\mathrm{Si}$ & 18003.757 & 4.87318 & 133.5381 \\
\hline Si-Si & 0 & 0 & 0 \\
\hline
\end{tabular}

The partial charges used are $q_{S i}=2.4$ and $q_{O}=-1.2$ with $e^{2}=14.3997 \mathrm{eV} \cdot \AA$. The original BKS potential does not specify the interaction between silicon atoms, nor cutoff for any of the interactions [1]. To get density of silica glass at room temperature closer to the experimental value and for better computational efficiency, a short-range cutoff of $5.5 \AA$ was later implemented [6].

The velocity Verlet algorithm was used to integrate the equations of motion with a timestep of 1.6 fs. Temperature was controlled by using a Nose-Hoover thermostat [23] and pressure with a Parrinello-Rahman barostat [24]. The long range Columbic interactions were evaluated by using the Ewald summation method [25] truncated at $10 \AA$. The short-range interactions were shifted and truncated at various cutoff values ranging from $5.5 \AA$ to $12 \AA$ to see the resulting structure and mechanical properties at ambient conditions and their changes under pressure at room temperature.

\subsection{Sample Preparation}

Glass samples were prepared by first taking a system of 10368 atoms to the liquid state around $7000 \mathrm{~K}$ in the NVT ensemble (constant number of atoms, volume and temperature) where it was equilibrated for around 200 ps. Samples were then brought down to around $5000 \mathrm{~K}$ in this ensemble and then subsequently quenched in the NPT (constant number of atoms, pressure, and temperature) ensemble to $300 \mathrm{~K}$ at a quench rate of around $5 \mathrm{~K} / \mathrm{ps}$. To understand the effect of the quenching rate, samples were also quenched at $0.5 \mathrm{~K} / \mathrm{ps}, 50 \mathrm{~K} / \mathrm{ps}, 200 \mathrm{~K} / \mathrm{ps}, 500 \mathrm{~K} / \mathrm{ps}$. For good statistics, four parallel samples were quenched starting from different times during the equilibration in the liquid state. Sample preparation was done using a short-range cutoff of $5.5 \AA$ to get a reasonable density of $2.24 \mathrm{~g} / \mathrm{cm}^{3}$ for silica glass at $300 \mathrm{~K}$. Quenched samples were relaxed at $300 \mathrm{~K}$ for $100 \mathrm{ps}$ by using different short-range cutoffs varying from $5.5 \AA$ to $12 \AA$.

For MD simulations under pressure, the samples were taken from $0 \mathrm{GPa}$ to $5.6 \mathrm{GPa}$ in steps of $0.8 \mathrm{GPa}$, the system was allowed to equilibrate at each pressure for around $30 \mathrm{ps}$. Mechanical tests were then performed on each of these samples at various pressures and averaged over the four parallel samples at each pressure. 


\subsection{Mechanical Test}

Bulk modulus was measured by first straining the sample hydrostatically in both the positive and negative direction to about $0.5 \%$ volume strain at a constant strain rate. The bulk modulus was then calculated around the equilibrium volume at each pressure by using the formula shown in Eq. (2).

$$
B=-V \frac{d P}{d V}=\frac{d P}{d \ln V}
$$

where $B$ is the bulk modulus, $V$ is the volume and $P$ is the pressure. Samples were also strained to $0.5 \%$ linear strain in one of the $x, y$ and $z$ directions at a time while keeping the cross-section constant. $\mathrm{C}_{11}$ was calculated from the slope of the stress vs. strain curve in a particular direction and averaged over multiple directions in four parallel samples. Poisson's ratio was also calculated from these simulations by using the equation shown in Eq. (3).

$$
\sigma_{1}=v\left(\sigma_{2}+\sigma_{3}\right)
$$

where $\sigma$ denotes the stress and 1,2,3 can represent $x, y$ and $z$ in any order. Other elastic moduli can then be calculated using any two of the three quantities calculated above.

To understand the structural contribution to the calculated mechanical properties, MD simulations were also carried out by relaxing a nearly ideal random-network model of amorphous silica in the BKS potential. This model has 1296 atoms with periodic boundary conditions, a density of $2.23 \mathrm{~g} / \mathrm{cm}^{3}$ at ambient conditions and each $\mathrm{Si}$ atom being 4-fold coordinated. This $a-\mathrm{SiO}_{2}$ model was originally obtained from a hand-built model for amorphous $\mathrm{Si}$ with $54 \mathrm{Si}$ atoms [26] by inserting O between the Si-Si bonds [27]. Over the years, this model was systematically improved, enlarged, fully relaxed using ab-initio methods and used as a representative model for $a-\mathrm{SiO}_{2}$ to calculate many different properties [18-21], including a recent study on amorphous to amorphous transition in silica under pressure [19] and in converting it to $a-\mathrm{GeO}_{2}$ to compare the properties between amorphous $\mathrm{GeO}_{2}$ and $\mathrm{SiO}_{2}$ [28]. In this study, the BKS quenched samples with a short-range cutoff of $5.5 \AA$ and a long-range cutoff of $10 \AA$ will be referred as BKS 5.5/10, those relaxed with both a short-range and a long-range cutoff of $10 \AA$ will be referred as BKS 10/10. The ab-initio sample relaxed in the BKS potential with a short-range cutoff of $5.5 \AA$ and $10 \AA$ and a long-range cutoff of $10 \AA$ will be referred as ab-initio 5.5/10 and ab-initio 10/10, respectively.

\section{Results}

\subsection{Cooling rate effect}




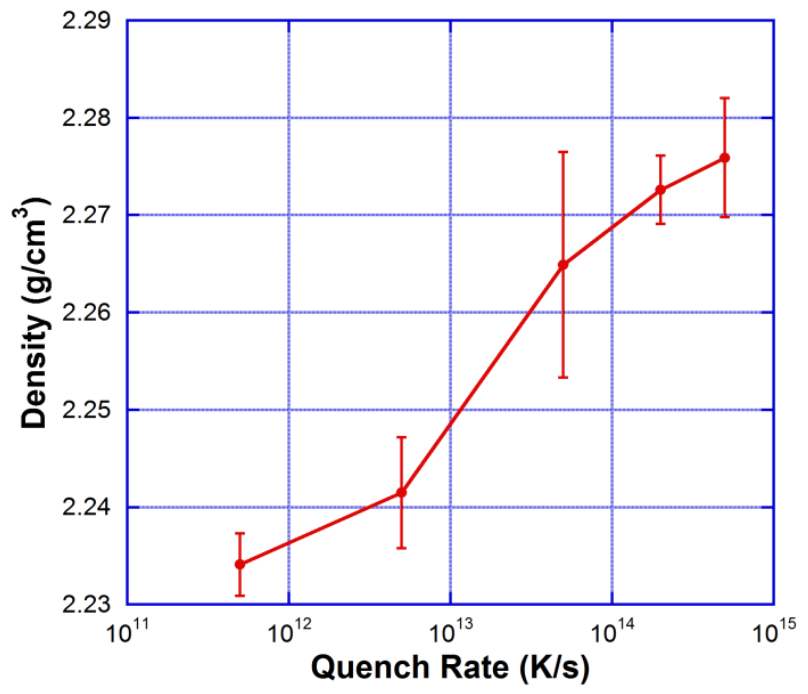

Fig. 1 Variation of density of quenched sample with the quench rate.

To understand the effect of quench rate, samples were first quenched to $300 \mathrm{~K}$ under different quench rates followed by relaxation by using the BKS potential with a short-range cutoff of 5.5 $\AA$ [6]. Fig. 1 shows the variation of density with the change of quench rate. Density tends to reduce and then saturate as the cooling rate reduces. Vollmayr et al. [6] performed a similar study by quenching under NPH (constant number of atoms, pressure and enthalpy) ensemble. The slight variations in our data compared to theirs can be attributed to the differences in the quenching method, but the trend remains the same. With the slowest quench rate, the modified BKS potential gives a density of $2.235 \mathrm{~g} / \mathrm{cm}^{3}$, which is close to the experimental value of 2.2 $\mathrm{g} / \mathrm{cm}^{3}$ for silica glass at ambient conditions [13].
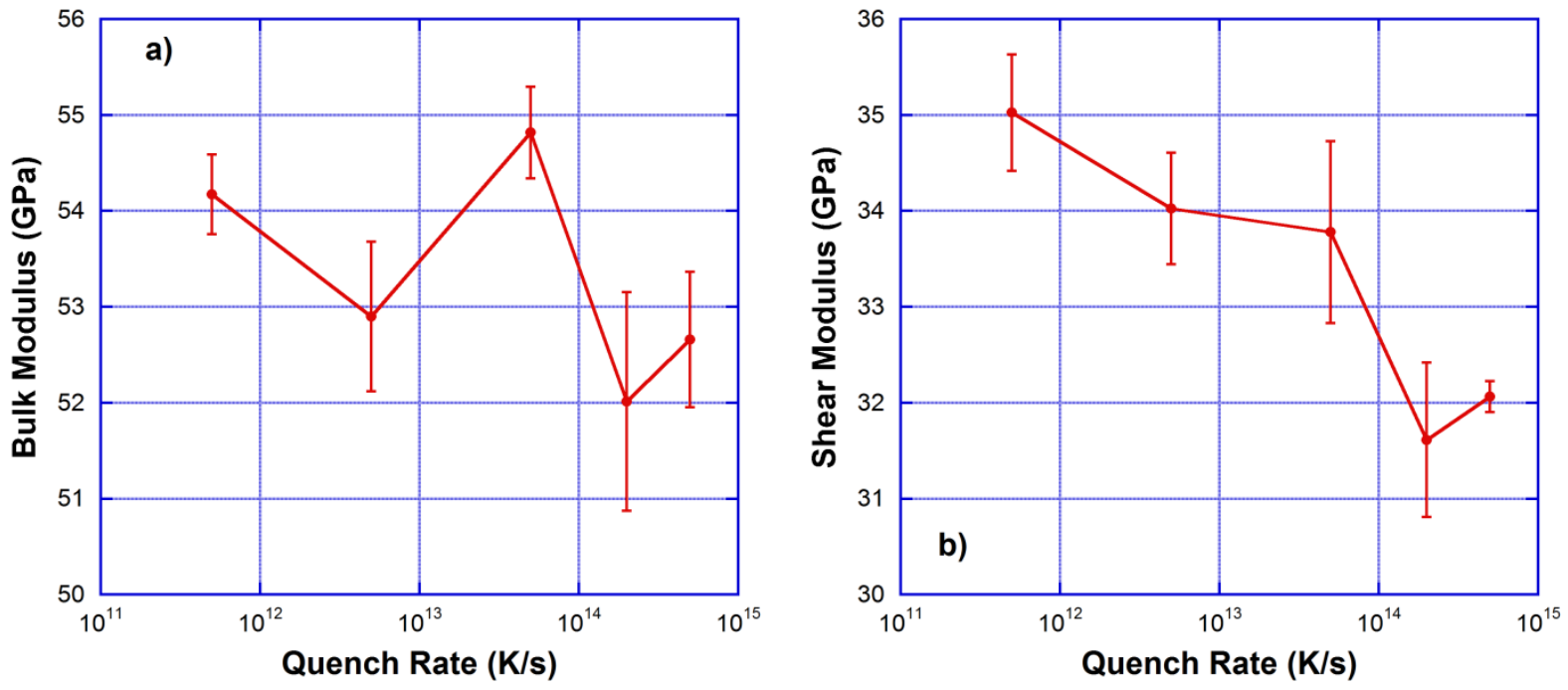

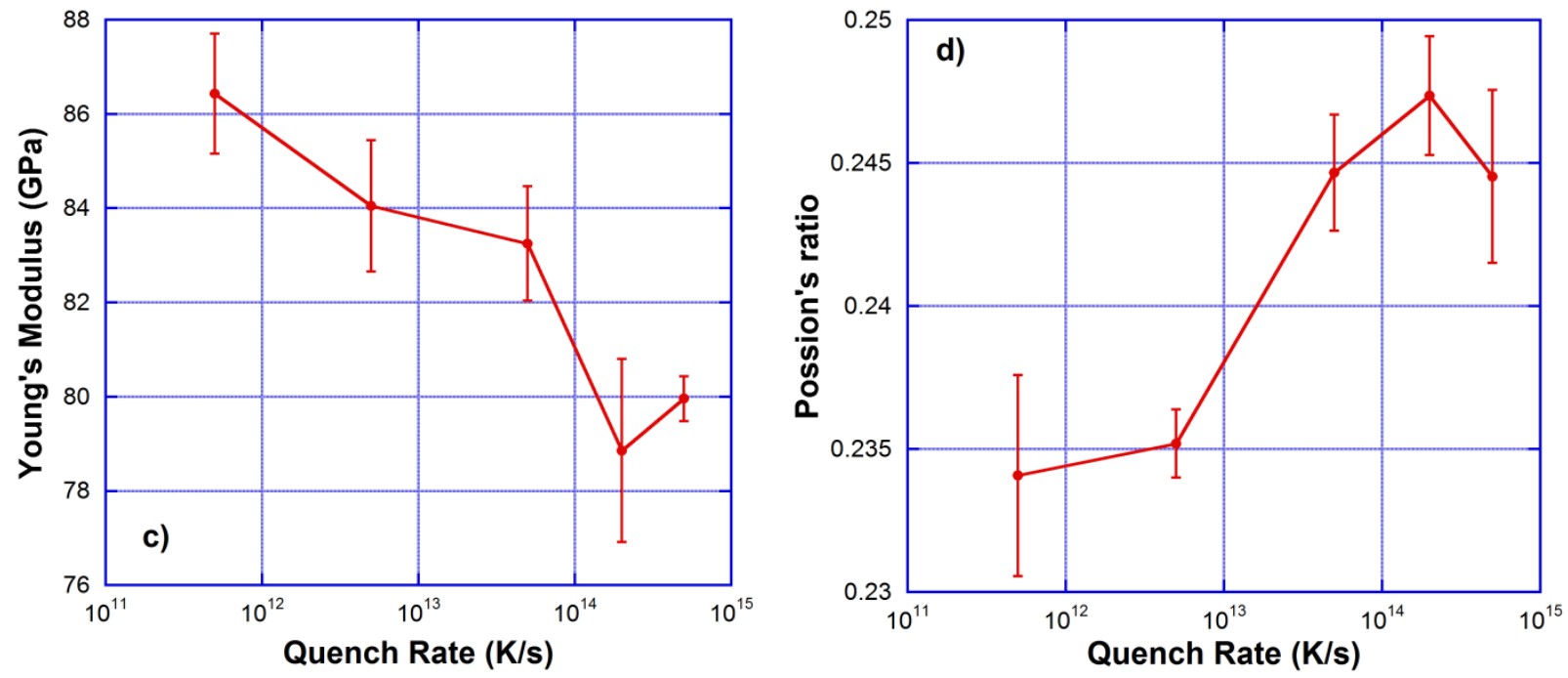

Fig. 2 Variation of a) bulk modulus, b) shear modulus, c) Young's modulus and d) Poisson's ratio with the quench rate.

Fig. 2 shows the variation of bulk modulus, shear modulus, Young's modulus and Poisson's ratio with the quenching rate. Bulk modulus increases slightly with the decrease of cooling rate but the change is very small, while shear and Young's modulus reduces with increasing cooling rate. Poisson's ratio also tends to reduce and then saturate as the cooling rate reduces. The predicted, bulk modulus, shear modulus, Young's modulus and Poisson's ratio are higher than experimental values [29], 54.2 vs. $36.4 \mathrm{GPa}, 35$ vs. $31.2 \mathrm{GPa}, 86.4$ vs. $72.7 \mathrm{GPa}$, and 0.234 vs. 0.17, respectively.

Elastic moduli were then measured under various pressures for samples prepared at various quench rates $(5 \mathrm{~K} / \mathrm{ps}, 50 \mathrm{~K} / \mathrm{ps}, 500 \mathrm{~K} / \mathrm{ps})$, and compared with experimental values [16] as shown in Fig. 3. Calculated elastic moduli are the average values from the four parallel samples and the error bars correspond to the standard deviations. It can be seen in Fig. 3 that for samples quenched under higher cooling rate, the elastic moduli except the Poisson's ratio are lower in the low pressure range. This is expected as the density and fictive temperature are higher and hence silica glass is softer [4]. It is also seen that the minimum in bulk, shear and Young's modulus move to lower pressures with increasing cooling rate. 

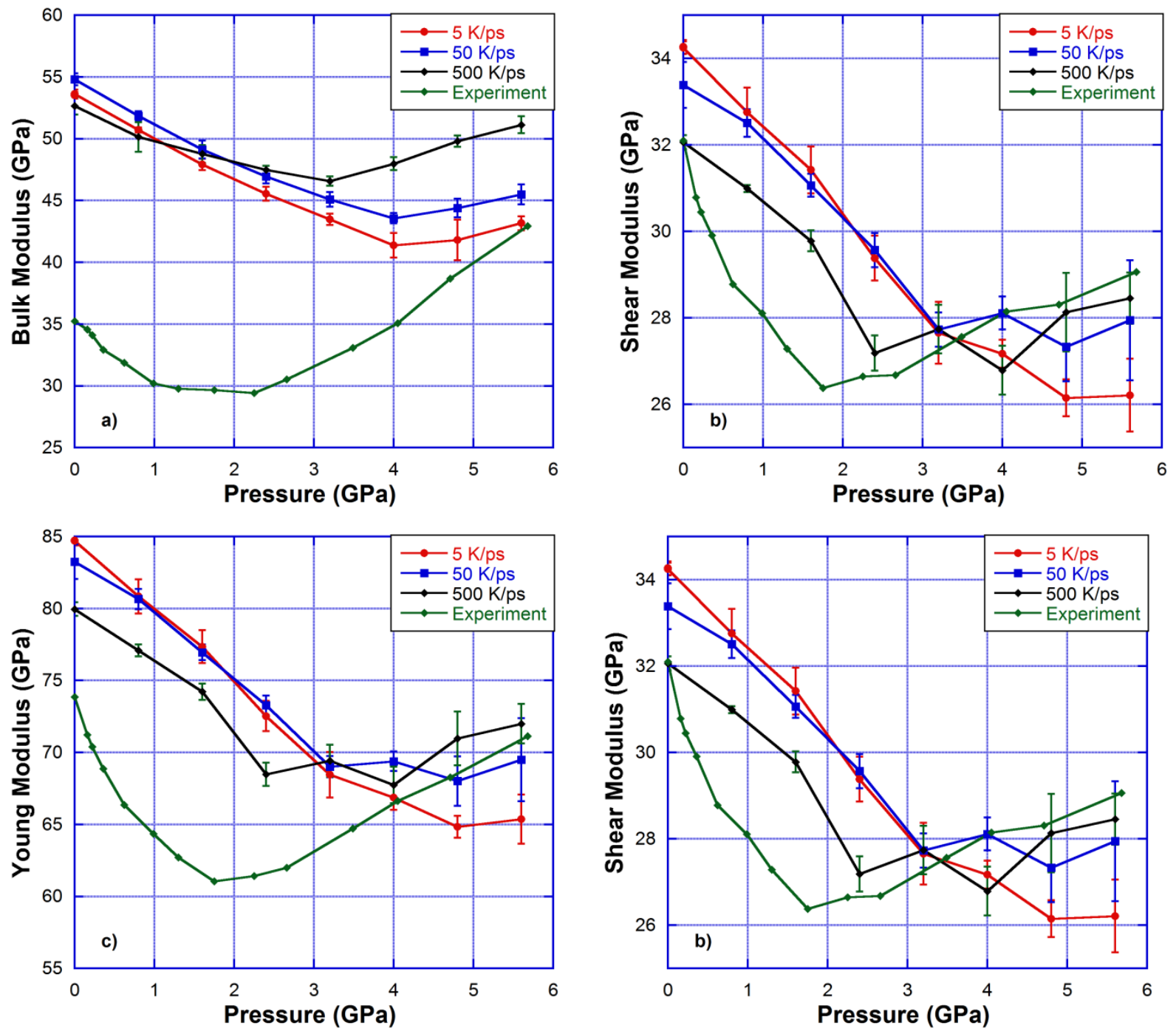

Fig. 3 a) Bulk modulus, b) shear modulus, c) Young's modulus and d) Poisson's ratio as a function of pressure for samples quenched under different cooling rates, experimental values are shown for comparison [16].

\subsection{Effect of short-range cutoff}




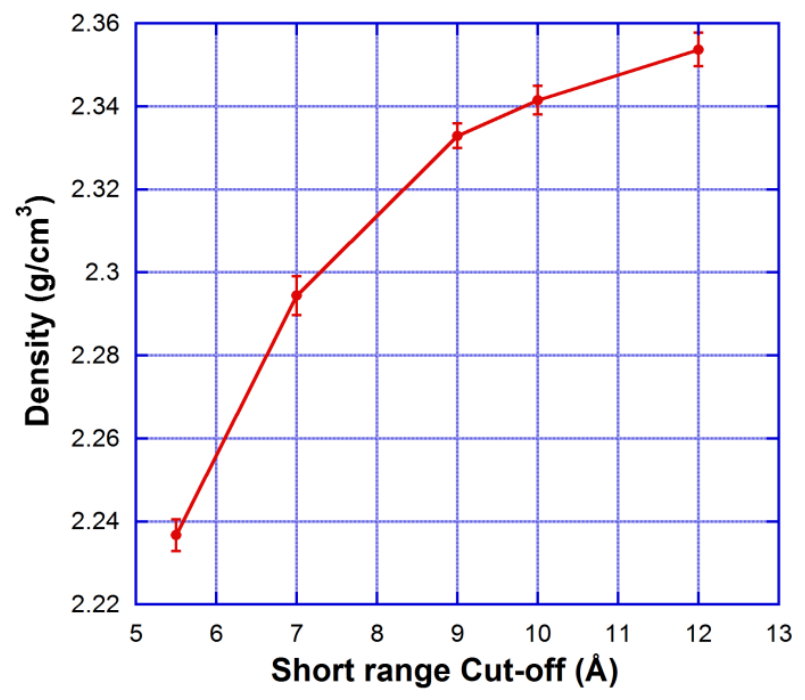

Fig. 4 Density of samples relaxed at various short-range cutoffs.

Samples were quenched under $5 \mathrm{~K} / \mathrm{ps}$ cooling rate and with a short-range cutoff of $5.5 \AA$, and then relaxed at $300 \mathrm{~K}$ using the various short-range cutoffs. Changes in density and elastic moduli with the change of the short-range cutoff are shown in Fig. 4 and Fig. 5, respectively. It can be seen from Fig. 4 that density increases by $\sim 5 \%$, while from Fig. 5 that bulk modulus, shear modulus and Young's modulus decreases by $\sim 26 \%, \sim 18 \%$ and $\sim 19 \%$, respectively, when the short-range cutoff increases from 5.5 to $12 \AA$., The Poisson's ratio shows a shallow minimum around a short-range cutoff of $10 \AA$, which was hence chosen for further study in this work. 

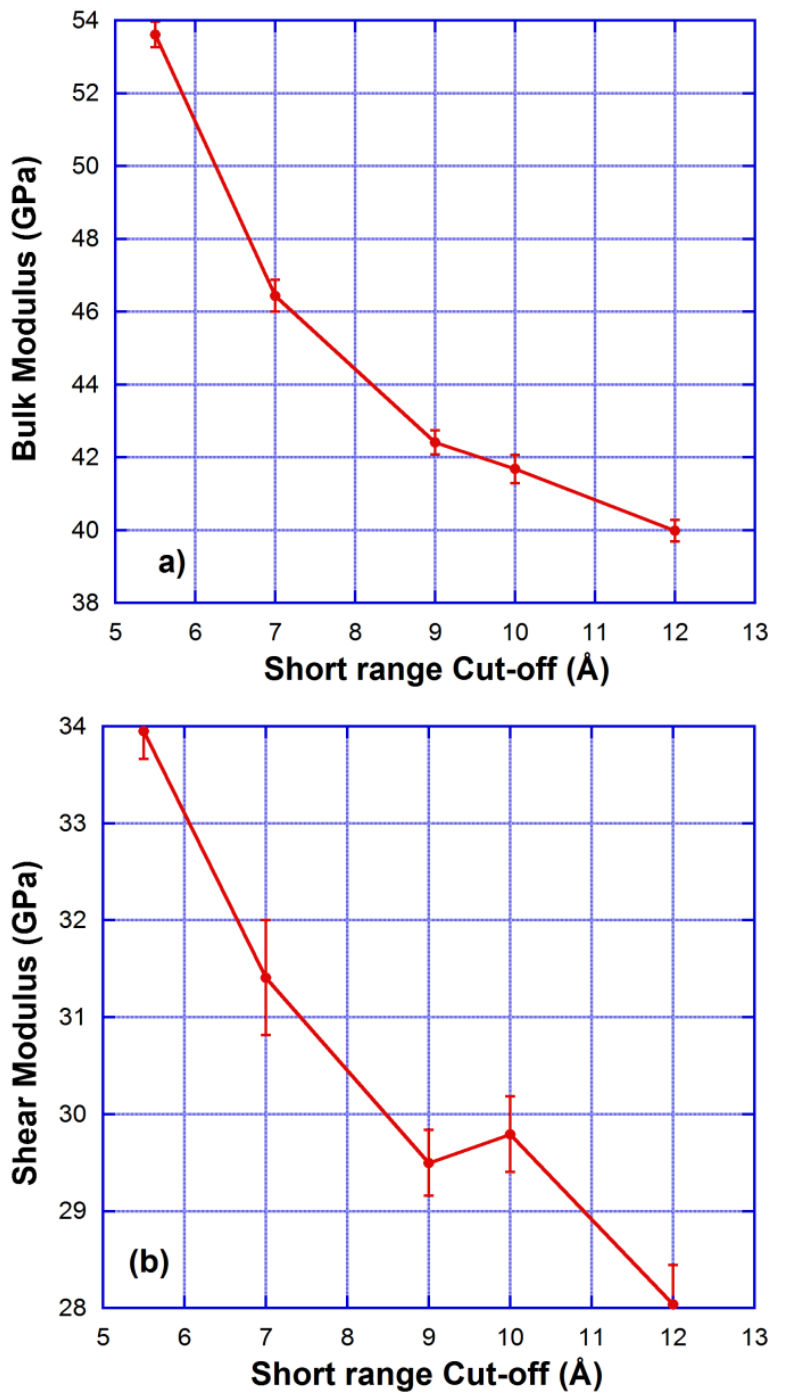

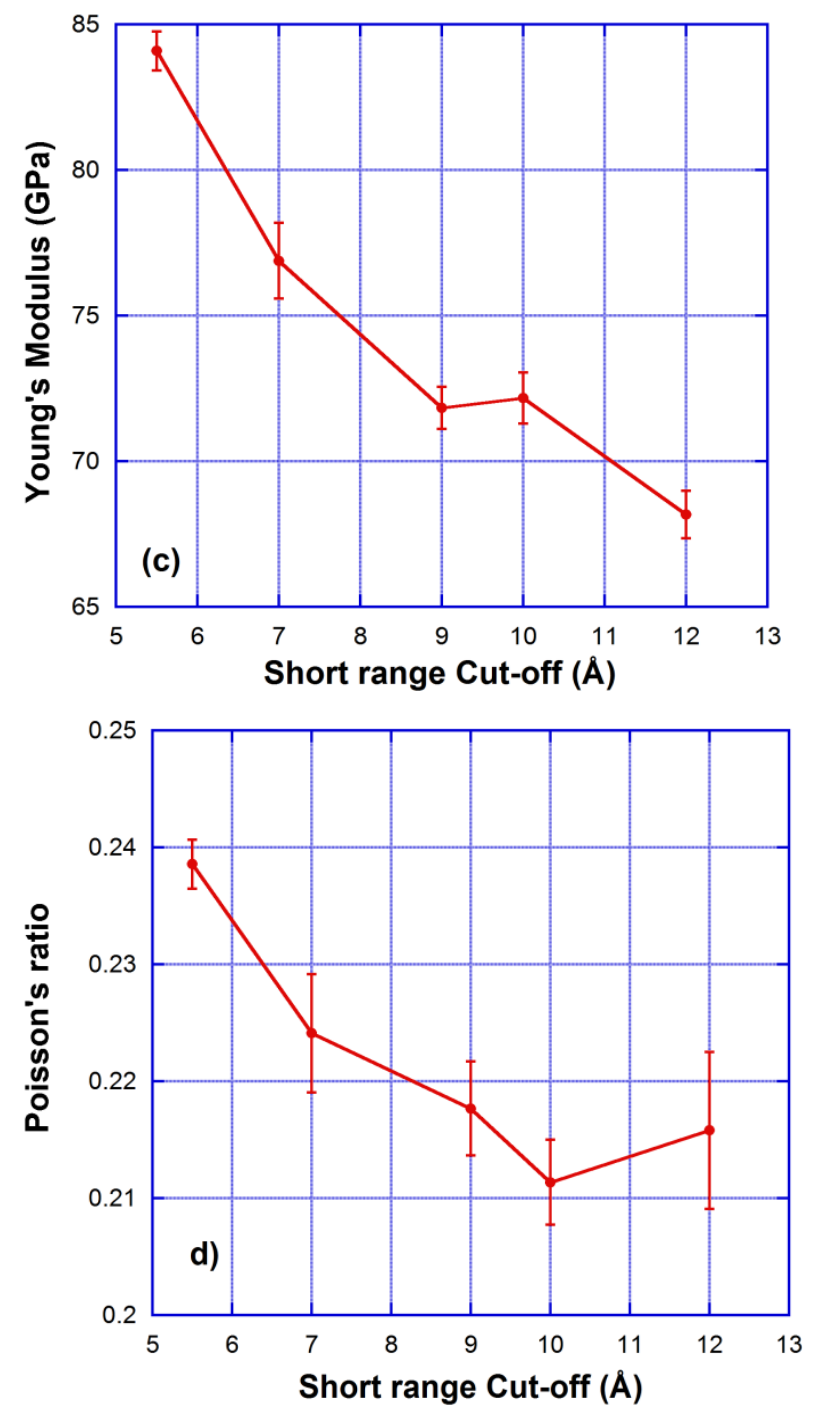

Fig. 5 a) Bulk modulus, b) shear modulus, c) Young's modulus and d) Poisson's ratio of samples relaxed at various short-range cutoffs.

Elastic moduli were then measured at $300 \mathrm{~K}$ under various pressures for the BKS 5.5/10 and BKS 10/10 samples and compared with experimental values [16] as shown in Fig. 6. Calculated elastic moduli are the average values from the four parallel samples and the error bars correspond to the standard deviations. It can be seen from Fig. 6 that for the higher short-range cutoff ( $10 \AA$ ), not only the values of mechanical properties at ambient conditions are closer to the experimental values, their changes with pressure at room temperature are also better predicted. For example, the well-known minimum in the bulk modulus as a function of pressure is predicted at $\sim 2.5 \mathrm{GPa}$, much closer to the experimental value of 2.2-2.3 $\mathrm{GPa}[13,16]$ than that predicted using the short-range cutoff of $5.5 \AA$. 

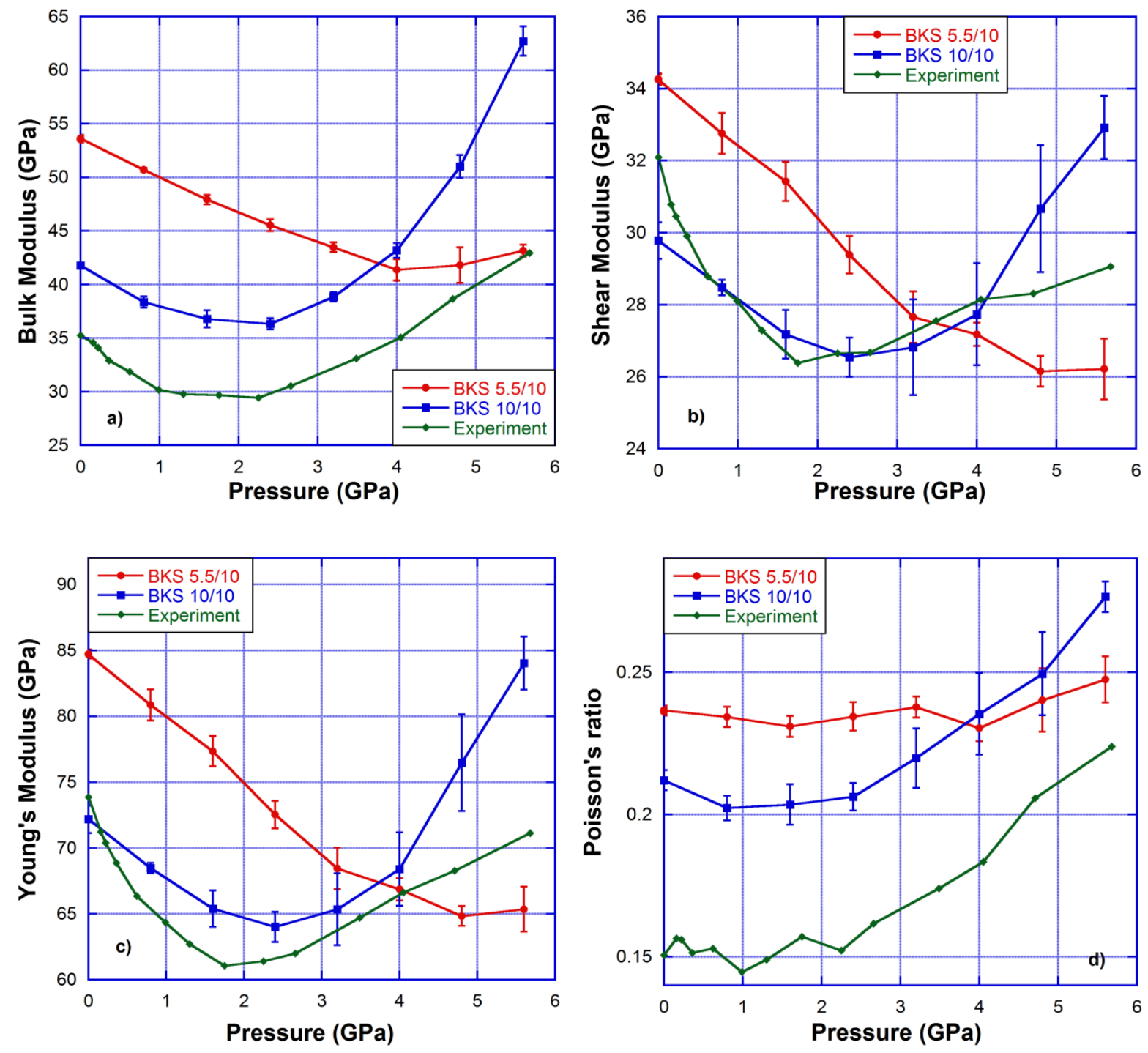

Fig. 6 a) Bulk modulus, b) shear modulus, c) Young's modulus and d) Poisson's ratio as a function of pressure for samples relaxed at various short-range cutoffs, experimental values are shown for comparison [16].

\subsection{Effect of structure}



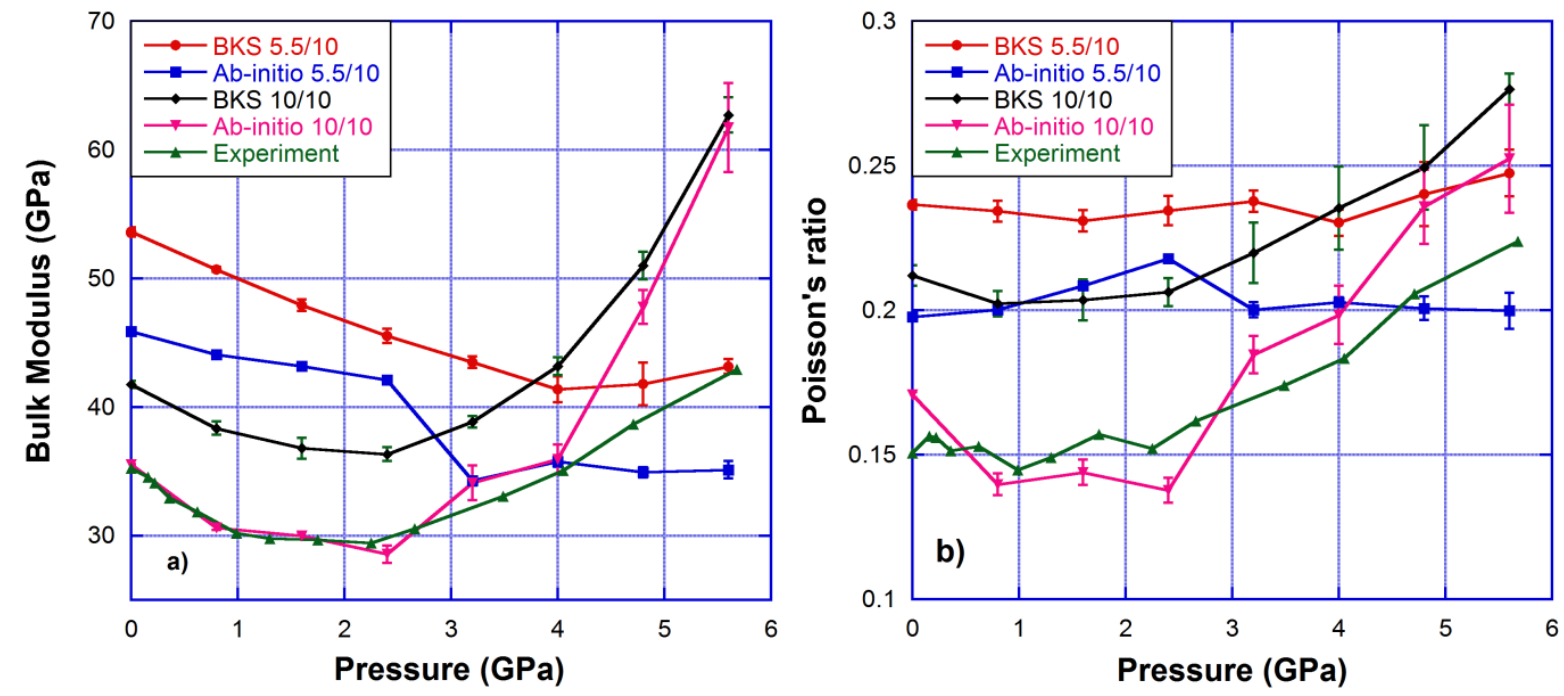

Fig. 7 Variation of a) bulk modulus and b) Poisson's ratio with pressure for various starting structures and short-range cutoffs, experimental values are shown for comparison [16].

To further understand the role of structure, the mechanical properties were calculated using a structure for amorphous silica created using ab-initio methods [18-21] as shown in Fig. 7. It can be seen from Fig. 7 that with the ab-initio structure, the mechanical properties at ambient conditions and their changes with pressure at room temperature are further improved by using the short-range cutoff of $10 \AA$. Especially for the Poisson's ratio, it is considerably reduced as compared with the BKS structure quenched using the short-range cutoff of $5.5 \AA$.

\section{Discussion}



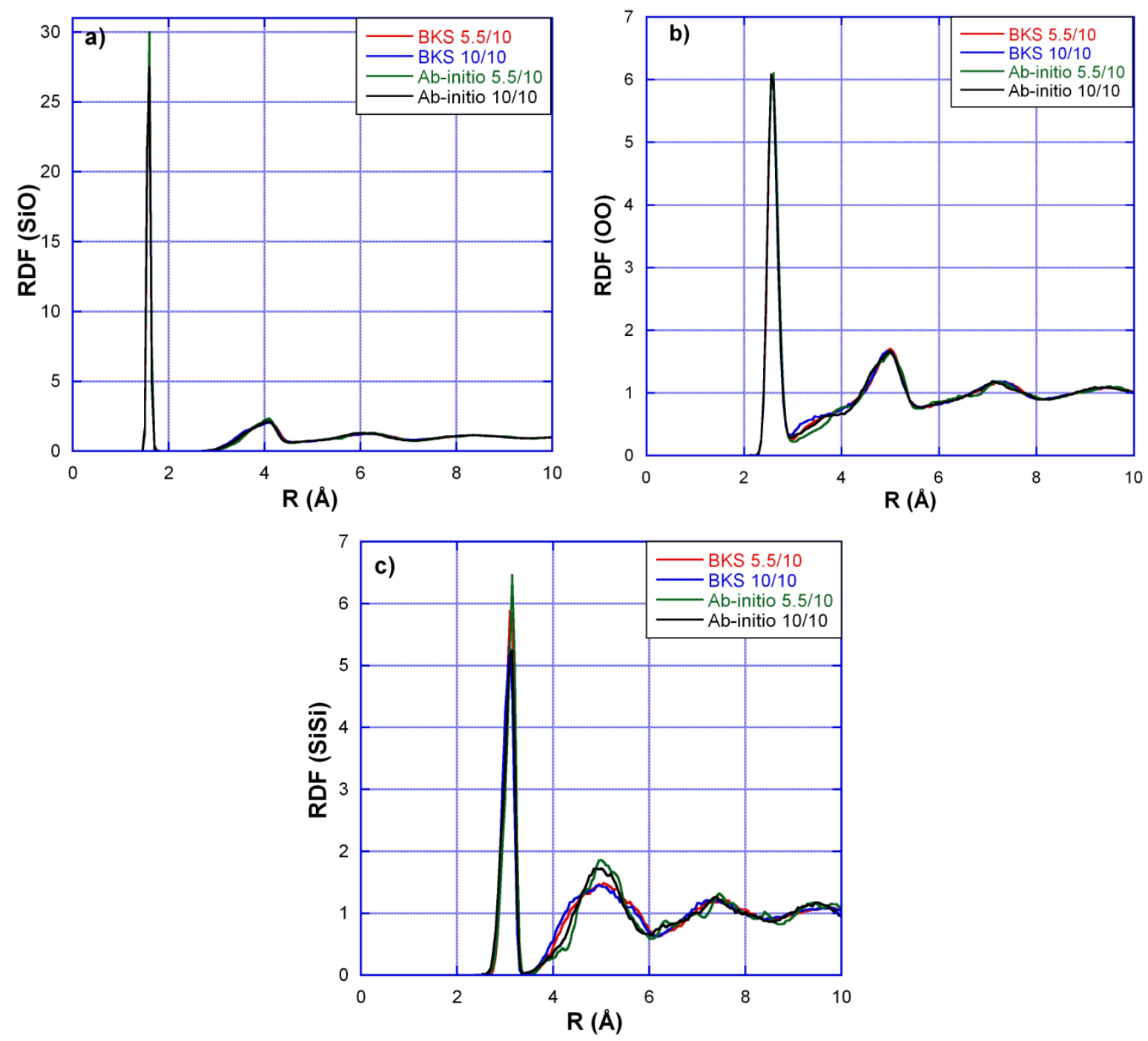

Fig. 8 a) Si-O, b) O-O and c) Si-Si radial distribution function. 

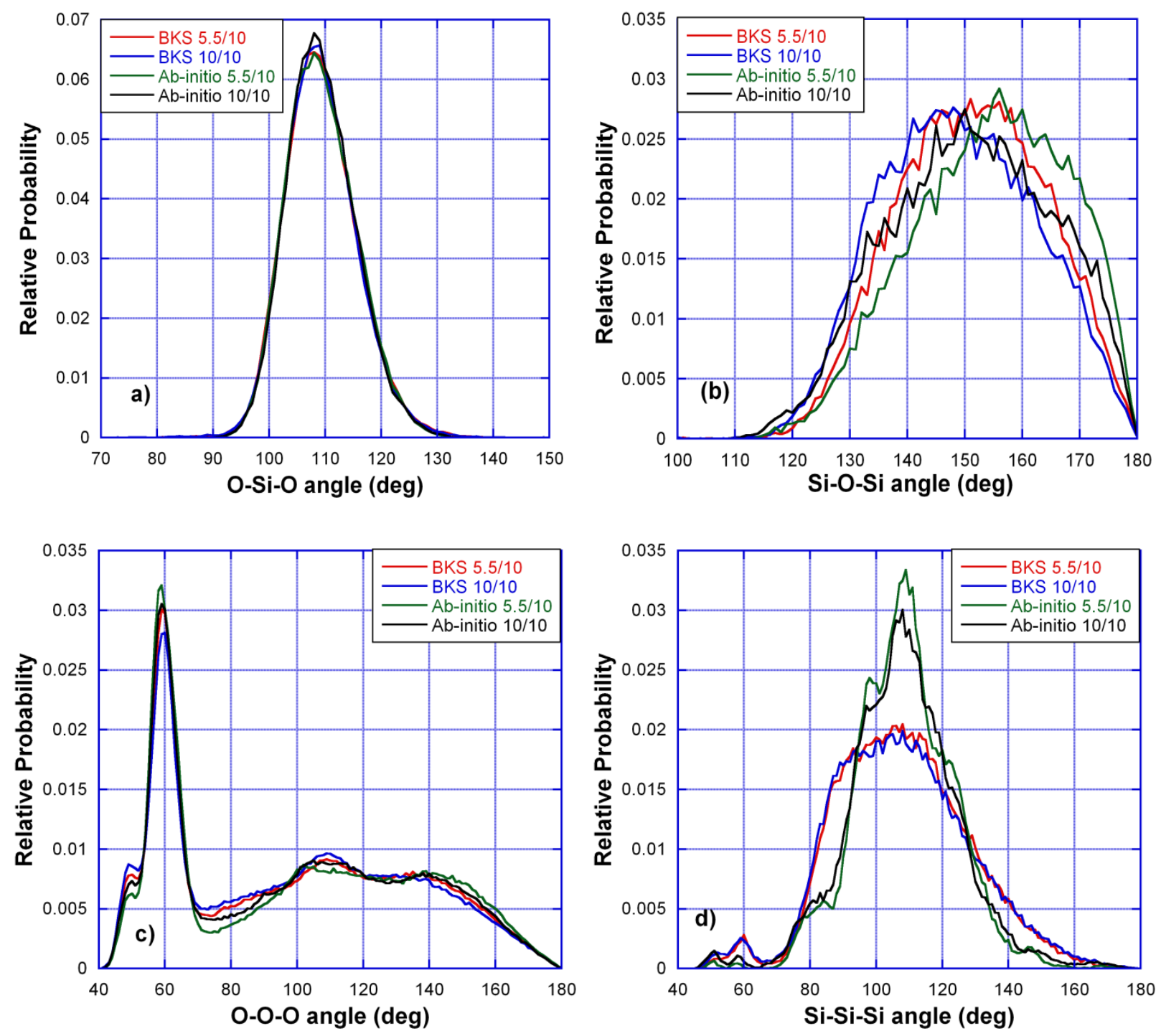

Fig. 9 Bond angle distribution for a) O-Si-O, b) Si-O-Si, c) O-O-O and d) Si-Si-Si triplets.

Structural changes due to the change in the short-range cutoff can be seen from the radial distribution functions (RDFs) in Fig. 8 and the bond angle distributions (BADs) in Fig. 9. The RDFs were calculated by binning the distances between various pairs into histograms and then normalizing with respect to the number density of an ideal gas. RDFs in Fig. 8 show no substantial change in the structure after relaxation with varying cutoff, except that the second Si$\mathrm{Si}$ peak is stronger in the ab-initio 5.5/10 and 10/10 than in the corresponding BKS ones. The intra-tetrahedral angle (O-Si-O) distribution shows no substantial change while the intertetrahedral angle ( $\mathrm{Si}-\mathrm{O}-\mathrm{Si}$ ) distribution shifts to lower angles with increasing short-range cutoff in agreement with the higher density seen in Fig. 4. Also, the higher intensity of Si-Si-Si BADs 
for the ab-initio samples in Fig. 9(d) show a greater medium-range order in agreement with the sharper second peak seen in the Si-Si RDF in Fig. 8(c).

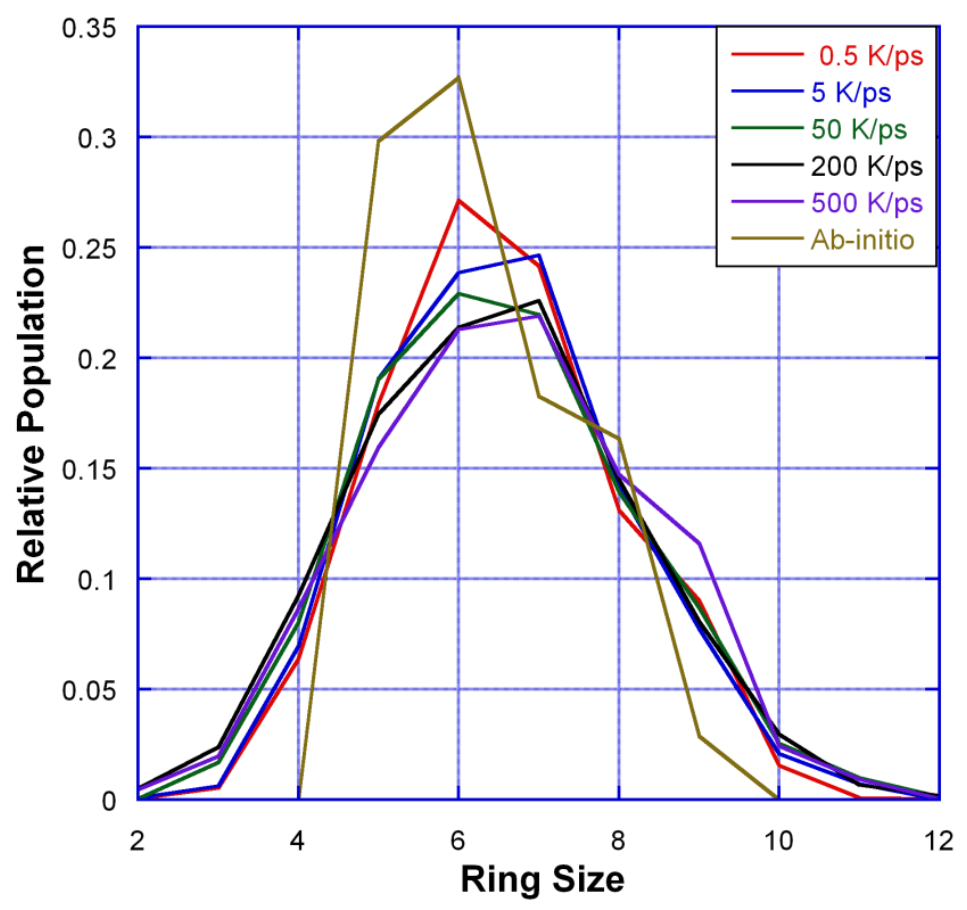

Fig. 10 Ring statistics for samples quenched under different cooling rates compared with the $a b$ initio structure.

Primitive rings [30,31] distribution was calculated using the R.I.N.G.S code [32]. The rings statistics in Fig. 10 show that as the quench rate is decreased, 6-membered rings become more popular while small rings (3- or 4- membered rings) are less, the ring size distribution gets closer to that in the ab-initio structure studied here and in a previous study [33]. It should be noted that there are no 3- or 4-membered in the ab-initio structure studied here, probably due to the specific way this structure was originally constructed [18-21]

Once a sample is quenched with certain cooling rate, the ring size distribution is seen to be identical before and after relaxation at room temperature using various cutoffs. In other words, the connectivity of the structure doesn't change during the relaxation process, the main change is seen in Si-O-Si BAD in Fig. 9(b). Table 2 summarizes some of the structural and mechanical properties of various samples in comparison with experimental values. 
Table 2. Structural and mechanical properties of samples calculated in this study in comparison with experimental values.

\begin{tabular}{|c|c|c|c|c|c|c|}
\hline Samples & $\begin{array}{c}\text { Density } \\
\left(\mathbf{g} / \mathbf{c m}^{\mathbf{3}}\right)\end{array}$ & Si-O $(\mathbf{A})$ & $\begin{array}{c}\text { O-Si-O } \\
(\mathbf{d e g})\end{array}$ & $\begin{array}{c}\text { Si-O-Si } \\
(\mathbf{d e g})\end{array}$ & Bulk Modulus (GPa) & Poisson's ratio \\
\hline BKS 5.5/10 & 2.24 & 1.615 & 109.4 & 151.1 & 53.4 & 0.24 \\
\hline BKS10/10 & 2.34 & 1.609 & 109.4 & 148.8 & 40.8 & 0.21 \\
\hline Ab-initio 5.5/10 & 2.14 & 1.615 & 109.4 & 154.3 & 46.7 & 0.19 \\
\hline Ab-initio 10/10 & 2.25 & 1.609 & 109.4 & 150.9 & 36.2 & 0.17 \\
\hline Experiments & $2.2[34]$ & $1.610[35]$ & $109.5[35]$ & $144[36]$ & $36.4[29]$ & $0.17[29]$ \\
\hline
\end{tabular}

A short-range cutoff of $5.5 \AA$ is usually chosen for the BKS potential as this gives a good quenched density for silica glass at ambient conditions. It can be seen from Fig. 8(b) that there is still strong O-O correlation well beyond the short-range cutoff of $5.5 \AA$, therefore, interactions at longer distances should have been included. This indicates that the correct density for silica glass quenched with a short-range cutoff of $5.5 \AA$ is likely resulted from error cancelling in the BKS potential. As-quenched density with a short-range cutoff of $10 \AA$ is very high $\left(\sim 2.50 \mathrm{~g} / \mathrm{cm}^{3}\right)$ as compared to the experimental value of $\sim 2.2 \mathrm{~g} / \mathrm{cm}^{3}$, so we chose to use a short-range cutoff of 5.5 $\AA$ for quenching and test the mechanical properties using various short-range cutoffs. Better prediction of mechanical properties is a further indication that the correct density for the sample quenched with a short-range cutoff of $5.5 \AA$ is just a coincidence. Figures 4 and 5 show that including the interactions at longer distances improves the prediction of mechanical properties at ambient conditions and their changes with pressure at room temperature.

The similarity in the structure between the original quenched structure and that relaxed with a higher short-range cutoff (Fig. 8 and 9) leads to the conclusion that the improvement in the mechanical properties is largely due to the addition of interactions at longer distances. Reducing the quench rate beyond a certain point does not further improve the density, mechanical properties or their changes under pressure (Fig. 1, 2 and 3). It was also seen that the improvement of BKS silica glass structure has a less effect in the correct prediction of the mechanical properties than the improvement of the interaction (Fig. 7). The above results indicate that either the interaction is more dominant in determining the mechanical properties of BKS silica glass or that the structure generated by the BKS potential is fairly good as suggested by Benoit et al. [9], which is the strength of this simple pair-wise potential. However, the 
problem that different short-range cutoffs are needed to get the correct as-quench density and mechanical properties is troublesome. A short-range cutoff is often chosen to reduce the computational cost, but should not influence the structure and properties substantially. This shows some fundamental flaws in the BKS potential model that should be addressed.

\section{Conclusions}

In this study, we have shown that increasing the short-range cut-off of the BKS potential improves both the mechanical properties at ambient conditions and their changes with pressure at room temperature. No major changes were observed in the structure in terms of radial distribution functions, bond angle distributions and ring statistics, after being relaxed using different short-range cutoffs. Further improvement in the mechanical properties was seen by using a better structure prepared by first principles methods. However, the effect of structure was shown to be less than that of the interaction potential in determining the mechanical properties of BKS silica glass. This either confirms that the BKS potential produces a decent structure or that the mechanical properties are more sensitive to the interaction potential. Future study is needed to fix the flaws in the BKS potential so that one short-range cutoff can be used to get the correct as-quenched density and other properties.

\section{Acknowledgements}

Liping Huang acknowledges the financial support from the National Science Foundation under grant No. DMR-1105238 and DMR-1255378. Wai-Yim Ching is supported by U.S. DOE, Office of Basic Energy Sciences, Division of Material Science and Engineering under Award DE-SC008176. This research used the resources of the National Energy Research Scientific Computing Center supported by DOE under Contract No. DE-AC03-76SF00098. Stimulating discussions with Drs. Walter Kob, Simona Ispas and Yunfeng Shi are greatly appreciated.

\section{References}

[1] G.J. Kramer, N.P. Farragher, B.W.H. Van Beest, R.A. Van Santen, Interatomic force fields for silicas, aluminophosphates, and zeolites: Derivation based on ab initio calculations, Phys. Rev. B. 43 (1991) 5068.

[2] D. Herzbach, K. Binder, M.H. Müser, Comparison of model potentials for moleculardynamics simulations of silica, J. Chem. Phys. 123 (2005) 124711.

[3] T.F. Soules, G.H. Gilmer, M.J. Matthews, J.S. Stolken, M.D. Feit, Silica molecular dynamic force fields - A practical assessment, J. Non-Cryst. Solids. 357 (2011) 1564-1573.

[4] F. Yuan, L. Huang, Molecular dynamics simulation of amorphous silica under uniaxial tension: From bulk to nanowire, J. Non-Cryst. Solids. 358 (2012) 3481-3487.

[5] F. Yuan, L. Huang, Brittle to ductile transition in densified silica glass, Sci. Rep. 4 (2014).

[6] K. Vollmayr, W. Kob, K. Binder, Cooling-rate effects in amorphous silica: A computersimulation study, Phys. Rev. B. 54 (1996) 15808. 
[7] I. Saika-Voivod, P.H. Poole, F. Sciortino, Fragile-to-strong transition and polyamorphism in the energy landscape of liquid silica, Nature. 412 (2001) 514-517.

[8] J. Horbach, W. Kob, Static and dynamic properties of a viscous silica melt, Phys. Rev. B. 60 (1999) 3169.

[9] M. Benoit, S. Ispas, P. Jund, R. Jullien, Model of silica glass from combined classical and ab initio molecular-dynamics simulations, Eur. Phys. J. B - Condens. Matter Complex Syst. 13 (2000) 631-636.

[10] J. Horbach, W. Kob, K. Binder, Specific Heat of Amorphous Silica within the Harmonic Approximation, J. Phys. Chem. B. 103 (1999) 4104-4108.

[11] P. Tangney, S. Scandolo, An ab initio parametrized interatomic force field for silica, J. Chem. Phys. 117 (2002) 8898-8904.

[12] C. Mischler, W. Kob, K. Binder, Classical and ab-initio molecular dynamic simulation of an amorphous silica surface, Comput. Phys. Commun. 147 (2002) 222-225.

[13] K. Kondo, S. Iio, A. Sawaoka, Nonlinear pressure dependence of the elastic moduli of fused quartz up to $3 \mathrm{GPa}$, J. Appl. Phys. 52 (1981) 2826-2831.

[14] J. Schroeder, T.G. Bilodeau, X.-S. Zhao, Brillouin and raman scattering from glasses under high pressure, High Press. Res. 4 (1990) 531-533.

[15] M. Grimsditch, Polymorphism in Amorphous $\mathrm{SiO}_{2}$, Phys. Rev. Lett. 52 (1984) 2379-2381.

[16] C. Weigel, A. Polian, M. Kint, B. Rufflé, M. Foret, R. Vacher, Vitreous Silica Distends in Helium Gas: Acoustic Versus Static Compressibilities, Phys. Rev. Lett. 109 (2012) 245504.

[17] B. Mantisi, A. Tanguy, G. Kermouche, E. Barthel, Atomistic response of a model silica glass under shear and pressure, Eur. Phys. J. B. 85 (2012) 1-13.

[18] N. Li, W.-Y. Ching, Structural, electronic and optical properties of a large random network model of amorphous $\mathrm{SiO}_{2}$ glass, J. Non-Cryst. Solids. 383 (2014) 28-32.

[19] N. Li, R. Sakidja, S. Aryal, W.-Y. Ching, Densification of a continuous random network model of amorphous $\mathrm{SiO}_{2}$ glass, Phys. Chem. Chem. Phys. 16 (2013) 1500-1514.

[20] M.-Z. Huang, L. Ouyang, W.Y. Ching, Electron and phonon states in an ideal continuous random network model of a-SiO 2 glass, Phys. Rev. B. 59 (1999) 3540-3550.

[21] M.-Z. Huang, W.Y. Ching, Electron states in a nearly ideal random-network model of amorphous $\mathrm{SiO}_{2}$ glass, Phys. Rev. B. 54 (1996) 5299-5308.

[22] S. Plimpton, Fast parallel algorithms for short-range molecular dynamics, J. Comput. Phys. 117 (1995) 1-19.

[23] W.G. Hoover, Canonical dynamics: equilibrium phase-space distributions, Phys. Rev. A. 31 (1985) 1695.

[24] M. Parrinello, A. Rahman, Polymorphic transitions in single crystals: A new molecular dynamics method, J. Appl. Phys. 52 (1981) 7182-7190.

[25] P.P. Ewald, Evaluation of optical and electrostatic lattice potentials, Ann Phys. 64 (1921) 253-287.

[26] L. Guttman, W.Y. Ching, J. Rath, Charge-Density Variation in a Model of Amorphous Silicon, Phys. Rev. Lett. 44 (1980) 1513-1516.

[27] W.Y. Ching, Microscopic Calculation of Localized Electron States in an Intrinsic Glass, Phys. Rev. Lett. 46 (1981) 607-610.

[28] B. Walker, C.C. Dharmawardhana, N. Dari, P. Rulis, W.-Y. Ching, Electronic structure and optical properties of amorphous $\mathrm{GeO}_{2}$ in comparison to amorphous $\mathrm{SiO}_{2}$, J. Non-Cryst. Solids. 428 (2015) 176-183. 
[29] M. Guerette, L. Huang, A simple and convenient set-up for high-temperature Brillouin light scattering, J. Phys. Appl. Phys. 45 (2012) 275302.

[30] K. Goetzke, H.-J. Klein, Properties and efficient algorithmic determination of different classes of rings in finite and infinite polyhedral networks, J. Non-Cryst. Solids. 127 (1991) 215-220.

[31] X. Yuan, A.N. Cormack, Efficient algorithm for primitive ring statistics in topological networks, Comput. Mater. Sci. 24 (2002) 343-360.

[32] S. Le Roux, P. Jund, Ring statistics analysis of topological networks: New approach and application to amorphous $\mathrm{GeS}_{2}$ and $\mathrm{SiO}_{2}$ systems, Comput. Mater. Sci. 49 (2010) 70-83.

[33] A. Pasquarello, R. Car, Identification of Raman Defect Lines as Signatures of Ring Structures in Vitreous Silica, Phys. Rev. Lett. 80 (1998) 5145-5147.

[34] M.H. Manghnani, B.K. Singh, Kunugi \& Tashiro \& Soga, in: Proc. Xth Int. Congr. Glass, 1974: p. 104.

[35] M.R. Vukcevich, A new interpretation of the anomalous properties of vitreous silica, J. Non-Cryst. Solids. 11 (1972) 25-63.

[36] E. Dupree, R.F. Pettifer, Determination of the $\mathrm{Si}-\mathrm{O}-\mathrm{Si}$ bond angle distribution in vitreous silica by magic angle spinning NMR, Nature. 308 (1984) 523-525. 